\title{
Datos corológicos y morfológicos de las especies del género Ambrosia L. (Compositae) presentes en la Península Ibérica
}

\author{
Ángel Amor Morales, Florentino Navarro Andrés y $\mathrm{M}^{\mathrm{a}}$ Angeles Sánchez Anta ${ }^{1}$
}

Resumen: Amor Morales, A.; Navarro Andrés, F. \& Sánchez Anta, Ma . A. 2012. Datos corológicos y morfológicos de las especies del género Ambrosia L. (Compositae) presentes en la Península Ibérica. Bot. Complut. 36: 85-96.

Se ha realizado un estudio corológico de las especies del género Ambrosia L. (Compositae) presentes en la Península Ibérica. Son Ambrosia maritima L., A. tomentosa Nutt., A. trifida L., A. artemisiifolia L., A. psilostachya DC. y A. tenuifolia Spreng. Además, utilizando material de herbario y ejemplares recolectados por los autores, se ha llevado a cabo un estudio morfológico y micromorfológico de las hojas y frutos de dichas especies, para lo que se ha utilizado el microscopio electrónico de barrido. Los datos obtenidos han permitido la elaboración de dos claves de determinación para estas especies, una basada en caracteres foliares y la otra en aspectos florales y carpológicos. El estudio corológico revela que la penetración de estos neófitos se produce a través de los litorales mediterráneo y atlántico, colonizando en primer lugar hábitats alterados por la actividad humana, como cunetas de carreteras, vías férreas, dunas marítimas, cultivos abandonados o riberas de ríos y arroyos.

Palabras clave: Ambrosia, corología, Compositae, claves de determinación, flora neófita, especies alóctonas, España.

Abstract: Amor Morales, A.; Navarro Andrés, F. \& Sánchez Anta, Ma . A. 2012. Chorological and morphological data concerning species of the genus Ambrosia L. (Compositae) present on the Iberian Peninsula. Bot. Complut. 36: 85-96.

A chorological study of the species of the genus Ambrosia L. (Compositae) present on the Iberian Peninsula has been performed. The species were Ambrosia maritima L., A. tomentosa Nutt., A. trifida L., A. artemisiifolia L., A. psilostachya DC. and A. tenuifolia Spreng. Additionally, using material from the herbarium and samples collected by the authors, we carried out a morphological and micromorphological study of the leaves and fruits of the species examined, in the latter case using a scanning electron microscope. The data obtained allowed the elaboration of two keys for the determination of these species; one based on leaf characteristics and the other on floral and carpological aspects. The corological study revealed that the penetration of the neophyte plants occurs through the Mediterranean and Atlantic littoral areas, first colonizing habitats altered by human activity, such as roadsides, railway lines, maritime dunes, abandoned crop lands and the banks of rivers and streams.

Key words: Ambrosia, chorology, Compositae, identification keys, neophyte flora, allochthonous species, Spain.

\section{INTRODUCCIÓN}

El género Ambrosia L. pertenece a la familia Asteraceae (Compositae) y está constituido por casi cuarenta especies originarias, la mayor parte, de Norteamérica y Sudamérica, desde donde se han propagado a otras áreas del globo, especialmente a Europa y Japón, en las cuales se consideran sinantrópicas, es decir, plantas ligadas a la acción voluntaria 0 involuntaria del hombre, que generalmente tiende a modificar su área de distribución natural por extensión (Sanz Elorza et al. 2004: 24)

El principal objetivo de este trabajo es dar a conocer un grupo de especies neófitas de dicho género, cuya presencia en la Península Ibérica pone de manifiesto el gra- ve problema que supone actualmente la introducción de seres vivos en territorios distintos a los de su área de distribución natural.

Suelen ser hierbas anuales, bienales o perennes, excepcionalmente arbustos, con tallos erectos, ramificados en la mitad superior, hojas alternas, opuestas o subopuestas, enteras, lobuladas (palmeadas) o profundamente divididas (bipinnatisectas), pecioladas o subsésiles, con peciolo alado o áptero. Partes aéreas de pubescentes a hirsutas. Las especies vivaces son estoloniferas.

Generalmente son monoicas, aunque Behçet (2004: 201-203) describe algunos individuos de la flora de Turquía correspondientes a Ambrosia tenuifolia Spreng. que producen solamente capítulos femeninos, hecho que rela-

\footnotetext{
${ }^{1}$ Departamento de Botánica, Universidad de Salamanca, Campus Miguel de Unamuno, s/n.37007 Salamanca.amor@usal.es, fna@usal.es, asanta@usal.es

Recibido: 28 septiembre 2011. Aceptado: 29 octubre 2011.
} 
ciona con la dioecia. Las flores se agrupan en capítulos pequeños e inconspicuos, unisexuados, los masculinos en inflorescencias racemosas 0 espiciformes terminales 0 axilares, los femeninos, situados por debajo de los masculinos, en la axila de brácteas o de hojas superiores reducidas, solitarios 0 en grupos de 2-3 0 incluso en glomérulos formados por más de tres capítulos. Brácteas del involucro soldadas, las de los capítulos masculinos herbáceas, las de los femeninos coriáceas. Capítulos masculinos discoideos, con flores flosculosas amarillas y receptáculo más o menos plano, pubescente, carente de páleas. Capítulos femeninos con una sola flor apétala por involucro, con excepción de A. tomentosa Nutt, cuyos capítulos femeninos son bifloros.

Polinización anemógama, favorecida por la disposición de los capítulos masculinos con respecto a los femeninos. El grano de polen es aeronavegante y el de algunas especies tales como A. trifida L. y A. artemisiifolia L., tiene una vida larga de permanencia en el aire y, sobre todo en el otoño, es responsable de serios trastornos de tipo alérgico en personas sensibles (Fireman 2007). Las cipselas (aquenios) carecen de vilanos y están totalmente envueltas por las brácteas del involucro. Este conjunto da lugar a una fructificación apiculada de sección poligonal, tan larga como ancha, o bien oblonga, de glabra a pubescente-glandulosa, con (4)5-7(8) costillas y otros tantos dientes espinosos o tuberosidades agudas, excepcionalmente algunas fructificaciones de A. psilostachya DC. son inermes. La dispersión de las diásporas es epizoócora.

La descripción de Ambrosia está basada en observaciones propias y en las de otros autores [cf. Bolós \& Vigo (1995: 769-771), Coste (1900-1906, 2: 481-482), Cronquist (1994, 5: 58), Gallego in Valdés et al. (1987, 3: 42), Jovet \& Vilmorin (1975, 3 supplèment: 299-300), Smoljaninova (1999, 25: 483-486), Tutin (1976, 4: 142-143)].

\section{MATERIALES Y MÉTODOS}

Para el estudio de la distribución geográfica del género $\mathrm{Am}$ brosia en la Península Ibérica se ha recurrido a revisar las citas de los principales herbarios existentes en el territorio. Concretamente se han consultado los herbarios BCN, FCO, HGI, JAEN, LEB, MA, MAF, MGC, SALA, SANT, SEV, VAL y VIT. También ha sido recolectado diverso material que ha quedado depositado en el herbario SALA de la Universidad de Salamanca. Asímismo, se han tenido en cuenta las citas recogidas en el programa Anthos desarrollado entre la Fundación Biodiversidad (Ministerio de Medio Ambiente) y el Consejo Superior de Investigaciones Científicas.
Para el estudio de los caracteres macro y micromorfológicos se ha utilizado tanto material fresco procedente de recolecciones llevadas a cabo por los autores, como material seco de los herbarios indicados. Se ha realizado con un microscopio estereoscópico al que se acopló una cámara fotográfica digital. También se han obtenido imágenes de los detalles microscópicos de hojas y frutos (pilosidad, tricomas, pústulas, etc.), utilizando un microscopio electrónico de barrido con sistema de microanálisis elemental por EDX.

\section{RESULTADOS}

En diversas publicaciones se ha señalado la presencia de varias especies del género Ambrosia en España y Portugal. Asimismo, existen numerosos pliegos de herbario que dan fe de la presencia de este género en la península. Se tiene constancia de la aparición de las seis especies siguientes: Ambrosia maritima, A. tomentosa, A. trifida, A. artemisiifolia, A. psilostachya y A. tenuifolia. En la Fig. 1 presentamos un mapa de distribución de las especies del género Ambrosia en la Península Ibérica. A continuación se comentan algunos aspectos taxonómicos, corológicos y bibliográficos de los taxones mencionados anteriormente.

\section{Ambrosia maritima L. (Figs. 2 y 5)}

Planta anual o bianual, con tallos erectos de hasta 50 $\mathrm{cm}$ y hojas inferiores bipinnatisectas discoloras, blancotomentosas en la cara abaxial. Hojas y tallos con pelos largos de base bulbosa con articulaciones poco netas, intercalados con excrecencias salinas, siendo más densos en la cara abaxial. Fructificación tan larga como ancha, pubescente glandulosa, provista de cinco costillas bien marcadas, 5(6) tuberosidades cónicas en la parte media y rematando en un pico con cinco pequeños dientes.

Linneo (1753: 988) en Species Plantarum II, cita esta especie en la antigua Etruria (Italia central) y Capadocia (Turquía) donde se desarrollaba sobre suelos arenosos marítimos. Diversos autores señalaron su existencia en España ya en el siglo XIX, como Willkomm \& Lange (1870: 274; 1893: 125), Boissier (1875: 252) o Pérez Lara (1887). Posteriormente ha sido citada en varias ocasiones por otros autores: Vayreda Vila (1901), Borja Carbonell (1951: 435), Montserrat (1962: 9), Folch Guillen (1976: 187).

Vive en dunas y arenales marítimos consolidados. Está distribuida por las costas del sur de Europa, Turquía, Egipto, Macaronesia (Cabo Verde) (Gallego in Valdés et al. (1987: 42), a diferencia de las otras especies estudiadas que son originarias de Norte y Sudamérica. 


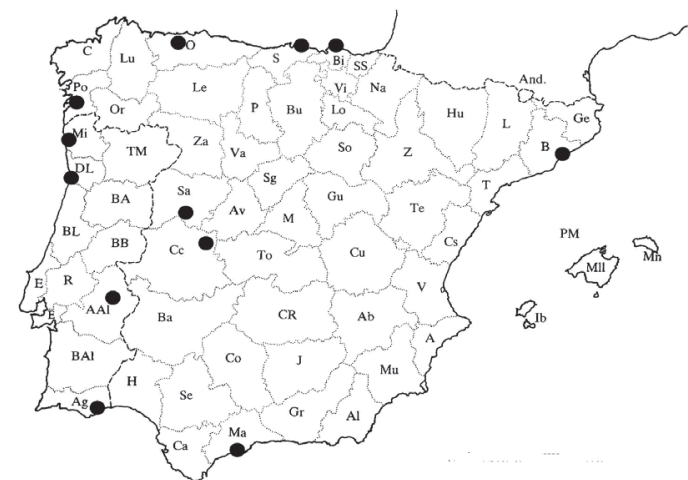

Ambrosia artemisiifolia

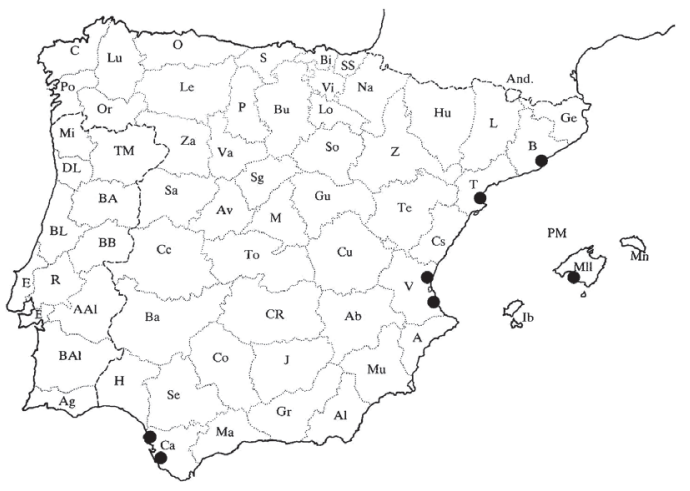

Ambrosia maritima

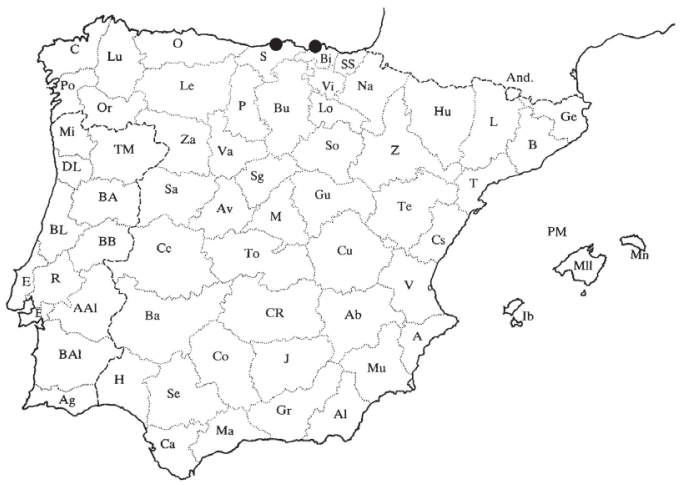

Ambrosia trifida

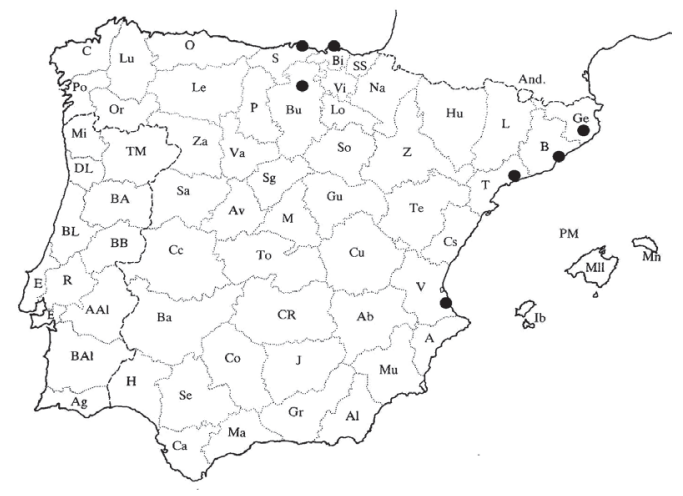

Ambrosia psilostachya

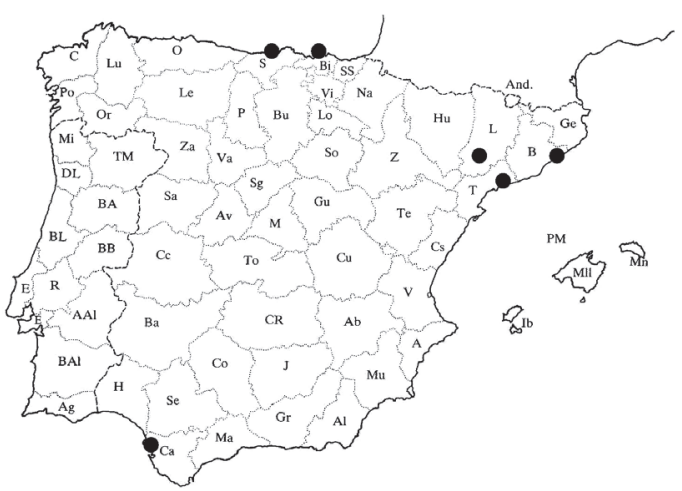

Ambrosia tenuifolia

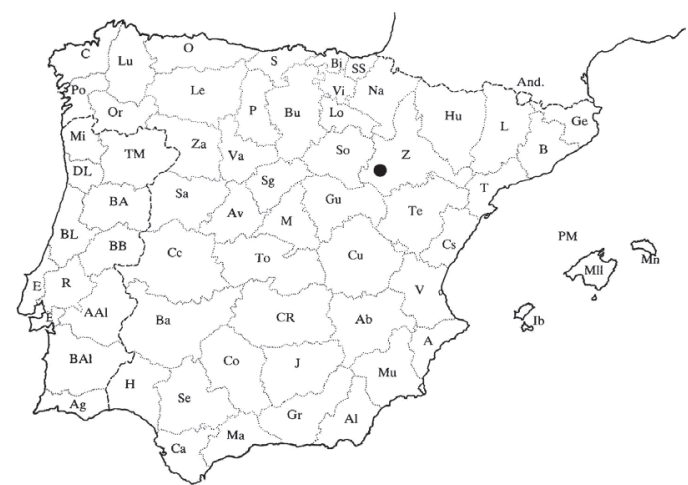

Ambrosia tomentosa

Fig. 1- Mapa de distribución de las especies estudiadas. 

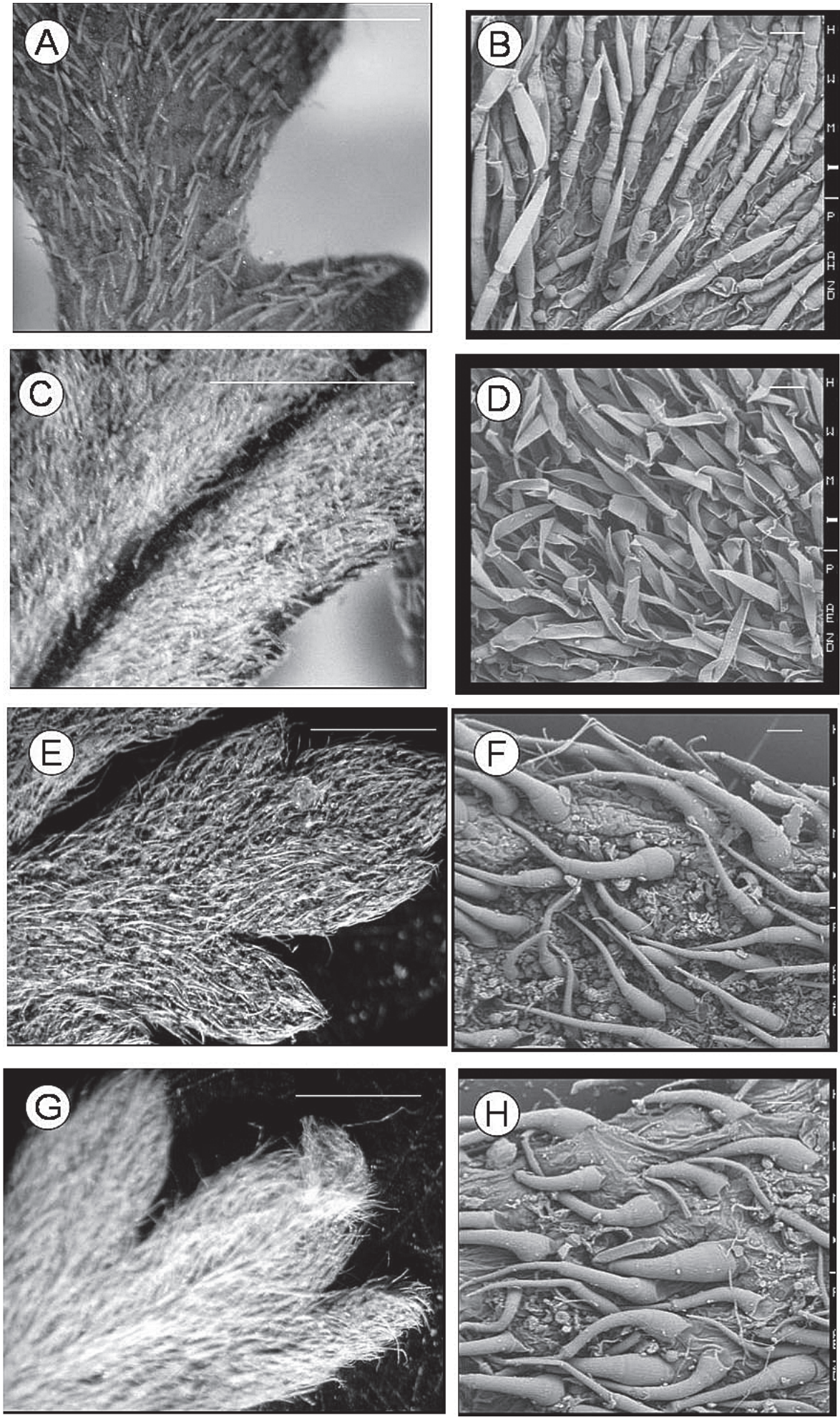

Fig. 2-Ambrosia tomentosa Nutt. A-B: cara adaxial de la hoja; C-D: cara abaxial de la hoja. Barra de escala: A-C: 1 mm; B-D: 50 $\mu \mathrm{m}$. A. maritima L. E-F: cara adaxial de la hoja; G-H: cara abaxial de la hoja. Barra de escala: E-G: $1 \mathrm{~mm} ; \mathbf{F}-\mathbf{H}: 50 \mu \mathrm{m}$. 
Se ha constatado su presencia en las provincias de Barcelona, Tarragona, Valencia, Cádiz y en la isla de Mallorca, como lo reflejan las citas bibliográficas anteriores y los siguientes pliegos de herbario: $\mathrm{CA}^{-}$ DIZ: Sanlúcar de Barrameda, 9-10-1981, S. Talavera \& B. Valdés, SALA 25737. Palma de Mallorca: Coll d'en Rabassa, X-1916, F. Bianor, BCN 31373. VALENCIA: Cullera, 30SYJ33, 2-VIII-1941, J. Borja, VAL 133049.

\section{Ambrosia tomentosa Nutt. (Figs. 2 y 5 )}

Especie vivaz, rizomatosa, de diez a cuarenta centímetros, hojas inferiores bipinnatisectas discoloras, con aspecto blanquecino por ser densamente tomentosas, cubiertas de pelos largos multicelulares con articulaciones bien visibles, con base bulbosa. Se diferencia del resto de las especies, que tienen involucro femenino unifloro $\mathrm{y}$ un solo aquenio, en que su involucro femenino es bifloro, con dos aquenios (cipselas); fructificación rematando en 2 picos, tomentosa, con pelos multicelulares dispersos.

Vive en bordes de caminos, cunetas y es originaria del centro y oeste de los Estados Unidos. En España sólo conocemos una cita de Calatayud (Zaragoza), donde fue recolectada por Segura Zubizarreta en 1986 (24 de mayo en flor, 21 de junio fructificada, como figura en la etiqueta del pliego. Herbarium A. Segura Zubizarreta, Soria, Hispania. $n^{\circ} 31542$ y 31543 ) y posteriormente distribuida con el número 13600 del Boletín no 22 de la Societè pour l'echange des plantes vasculaires de l'Europe et del bassin mediterraneen (Lambinon 1989). Sanz Elorza et al. (2009: 74) también mencionan esta presencia en Zaragoza como planta casual introducida involuntariamente.

\section{Ambrosia trifida L. (Figs. 3 y 5)}

Esta especie se denomina vulgarmente ambrosia gigante y es una planta anual que puede superar fácilmente el metro de altura, con tallo erecto y hojas opuestas, palmeadas, divididas en tres o cinco lóbulos lanceolados, acuminados y finamente serrados en los bordes, escabras, pustulosas y pubescentes por ambas caras, con pequeños pelos estrigosos acompañados de otros cortos unicelulares dispersos, que en la cara abaxial, se localizan fundamentalmente en los nervios. Capítulos masculinos con involucro cupuliforme, un poco asimétrico, glabro, con algunos pelos multicelulares cortos y rígidos de base bulbosa. Capítulos femeninos agrupados en glomérulos axilares. Involucro femenino con (4-5) 7 tuberosidades situadas en la mitad superior de la fructificación, la cual es oblonga y glabra, con algunos pelos pluricelulares en la parte apical que remata en un pico cónico.

Como otros congéneres, es una planta nitrófila que se comporta como mala hierba de cultivos; también aparece en bordes de caminos, escombreras y barbechos. Es originaria de Norteamérica y se halla introducida en varios países europeos. En España las citas que se conocen son del norte del territorio. Laínz \& Loriente (1983: 412) señalan su presencia en Santander (Cantabria).

También hemos revisado material de herbario recolectado en la provincia de VIZCAYA: Santurce, 30TVN9697, 5-X-1996, S. Patino \& J. Valencia, VIT 54280. Portugalete, 30TVN9895, 15-IX-1995, S. Patino \& J. Valencia, VIT 75496.

\section{Ambrosia artemisiifolia L. (Figs. 3 y 5)}

Planta anual con hojas subsésiles en su mayoría, las inferiores con un corto peciolo alado, con algunos pelos multicelulares estrigosos, cortos, de base bulbosa, intercalados con tricomas simples más o menos glandulares. En la cara abaxial, los pelos cortos se entremezclan con otros de mayor tamaño e idénticas características. Capítulos masculinos turbinados, pubescentes y ciliados, con pelos multicelulares cortos de base bulbosa, entremezclados con pelos largos. Capítulos femeninos agrupados en glomérulos axilares. Involucro fructífero oblongo, con (4) 5-6 (7) dientes espinosos, ubicados en su mitad superior, provisto de tricomas glandulares simples intercalados con algunos pelos multicelulares y que remata en un pico provisto de cinco pequeños dientes.

Es un neófito (metáfito, epecófito/hemihagrófito) de origen americano que fue introducido en Europa hacia 1865. En la actualidad es una planta alóctona invasora en gran parte de Europa, en algunas zonas de Asia, América Central y Caribe, América del Sur, Japón y Australia (Sanz Elorza et al. 2001: 128; 2004: 78).

Este taxón en la Península Ibérica suele aparecer en medios antropizados, como bordes de caminos, suelos removidos, cultivos abandonados, orillas nitrificadas de ríos y arroyos. Las primeras citas en la Península Ibérica se deben a Pinto da Silva (1972: 24), quien la recolectó en Montemor-o-Novo (Alto Alentejo, Portugal). Amaral Franco (1984: 384) señala nuevas localidades del NW de Portugal. En España ha sido citada por autores como Lainz \& Loriente (1983: 411) en Santander (Cantabria), García Martínez (1985: 192) en Pontevedra (Galicia), Trigo \& García-Sánchez (2006: 203) en Málaga (Andalucía), Amor et al. (2007: 133) en las provincias de Salamanca y Cáceres. 

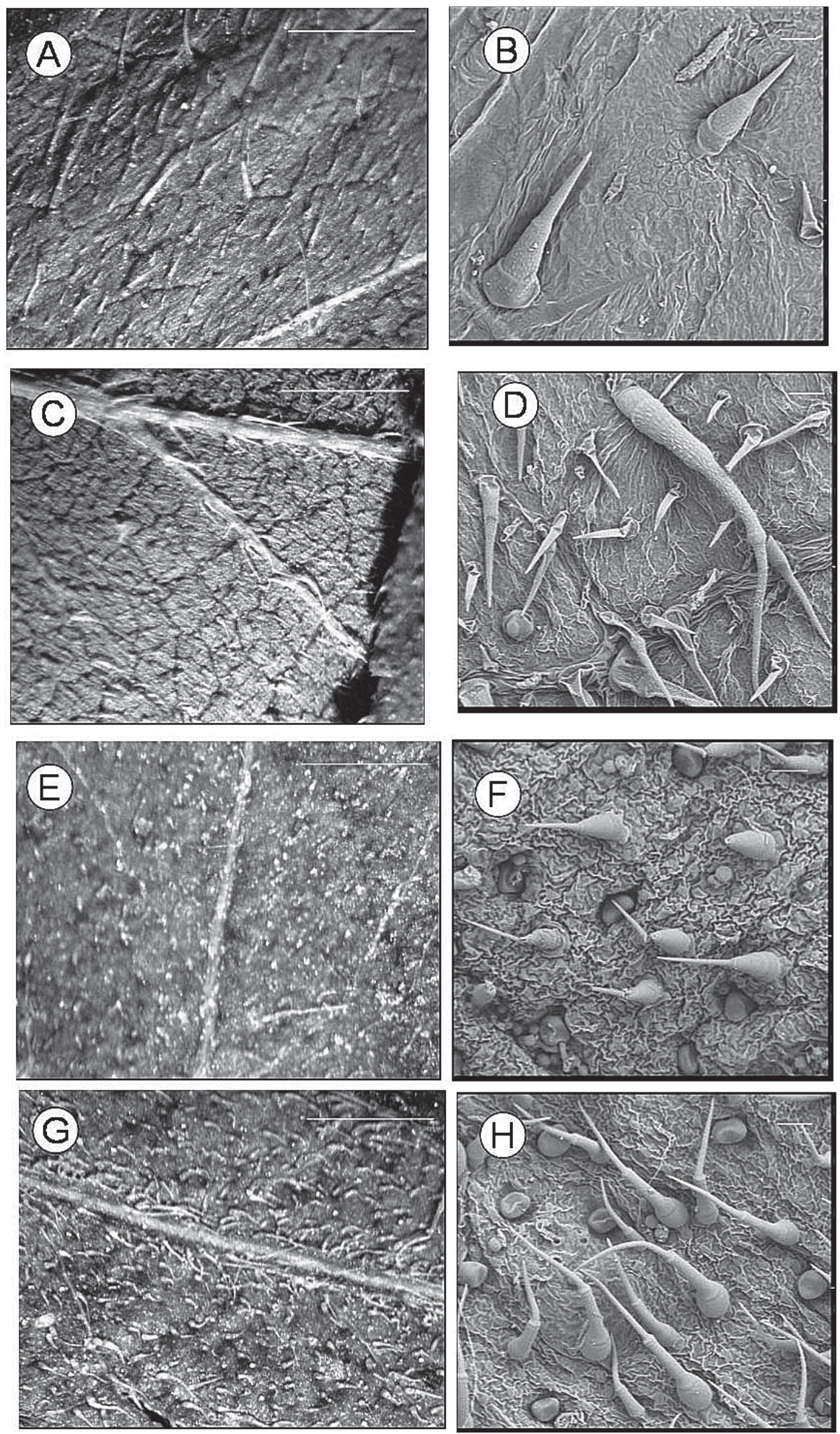

Fig. 3-Ambrosia trifida L. A-B: cara adaxial de la hoja. C-D: cara abaxial de la hoja. Barra de escala: A-C: $1 \mathrm{~mm}$; B-D: $50 \mu \mathrm{m}$. A.

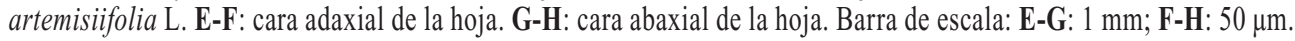


Aparte de las citas bibliográficas hemos consultado diverso material: Asturias: Salas, pr. La Barrosa, 29TQJ2208, 23-X-1997, H. S. Nava, FCO 24.612. BARCELONA: El Corredor (Maresme), 8-VI-1982, Perdigó \& Mait, BCN 20550. CÁCERes: Majadas de Tiétar, 30STK6626, 12-IX-2004. A. Amor, SALA 108748. CanTABRia: Santander, 30TVP31, 6-IX-1982, Aedo, Loriente \& Patallo. Santoña, 30TVP6212, 3-VIII-1984, J.A. Fdez. Prieto \& M. Herrera, SANT 21686. CoRuÑa: Rianxo, 18-IX1996, V. Rial Ces, SANT 44001. Pontevedra: Raxó, playa de Sinás, 29TNG2094, 30-IX-1984, X. R. Garcia Martínez, SANT 13686. 0 Grove, playa Buraco da Londra, 29TNG0701, VIII-1984, X. R. García Martínez. Salamanca: Valero, 30TTK4491, 3-IX-2004, F. Navarro, SALA 110589. VIZCAYA: Ortuella, 30TVN9795, 15-X-1997, J. A. Campos, MAF 158330. Santurce, 30TVN9695, 4-X-1994, J. Elorza \& S. Patino, VIT 75.485. Portugalete, 30TVN9795, 6-X-1995, S. Patino \& J. Valencia, VIT 75482. Zorroza, 30TWN0292, 2-X-1996, S. Patino, VIT 75480.

\section{Ambrosia psilostachya DC. (= A. coronopifolia} Torr. \& A. Gray) (Figs. 4 y 5 )

Planta vivaz rizomatosa, con tallos erectos, hojas pinnatipartidas, subsésiles o con un corto peciolo alado, con tomento de pelos aplicados y con pústulas de morfología similar a las de A. artemisiifolia. Fructificación oblonga, de pubescente glandulosa a glabra, con cuatro tuberosidades muy cortas, excepcionalmente inerme; los dos tercios superiores cubiertos con tricomas uni y multicelulares.

Ambrosia psilostachya aparece en medios ruderalizados como bordes de caminos, terrenos baldíos, orillas pedregosas de ríos y arroyos, etc. Es una planta sinantrópica (metáfito, hemiagriófito) distribuida originariamente por América Central y Norteamérica. Se encuentra naturalizada en varios países de Europa, entre ellos España. Se ha citado de las siguientes provincias: Tarragona (Rico Hernández 1981: 308), Gerona (Vallés Xirau \& Poch Ymbert 1999), Valencia (Carretero 1990) y Cantabria (Segura Zubizarreta 1982). Sanz Elorza et al. (2001: 129) la consideran planta invasora en España.

Se ha comprobado que algunos de los pliegos consultados de esta especie están mal identificados. Concretamente, los pliegos VIT 57060, VIT 57100 y VIT 57104, con material recolectado en Portugalete (Vizcaya); el pliego VIT 57099, procedente de Zorroza (Vizcaya), y el pliego SANT 17691, de Santoña (Cantabria), pertenecen en realidad a la especie Ambrosia artemisiifolia.

Asimismo, hemos consultado citas de herbario que demuestran su presencia en Barcelona y Burgos: BarCELONA: Montcada i Reixac, 31TDF3090, 13-X-1985, Benedi, SALA 45394. Montmeló, 31TDG30, 6-X-1981, Casasayas \& Forcadell, BCN 31377. Burgos: Miranda de Ebro, 30TWN0426, 28-IX-1996, J. Elorza, VIT 75492. Gerona: La Selva, 31TDG73, 5-IX-1992, J. Vallés Xirau, BCN16253. CantabRia: Santoña, 27-VII-1985, J. C. Fdez. de la Mela, SANT 17691. TARRAGONA: Segur de Calafell, 2-VIII-1976, E. Rico, SALA 13155.

\section{Ambrosia tenuifolia Spreng. (Figs. 4 y 5 )}

Planta vivaz rizomatosa, estolonífera, hojas opuestas, pecioladas, bipinnatisectas y con lóbulos lineares. Tomento foliar semejante al de A. psilostachya, pero más denso. Capitulos femeninos generalmente solitarios, raramente geminados. Fructificación oblonga, pubescente-glandulosa, con pelos multicelulares y tricomas glandulares simples, con (4) 5-6 (7) dientes espinosos, rematando en un pico corto con cinco dientes.

Tiene un comportamiento ruderal y viario, se desarrolla en taludes, bordes de caminos y dunas marítimas algo nitrificadas. Originaria de América del Sur, naturalizada en España, Francia e Italia (metáfito epicófito), fue citada por primera vez en España por Montserrat (1954), quien la recolectó en Llinars del Vallès (Barcelona), sobre suelos gravosos próximos a la vía férrea. Posteriormente ha sido señalada por otros autores como Gallego \& Valdés (1984: 270), quienes la hallaron en Andalucía, concretamente en el Puerto de Santa María (Cádiz). Masalles et al. (1996: 78) encontraron la planta en las provincias catalanas de Lérida y Tarragona. Verloove (2005: 142) la cita de Montmeló (Barcelona) en el lecho gravoso del río Besós. Sanz Elorza et al. (2001: 129) la señalan en la lista preliminar de plantas con comportamiento invasor incipiente en España.

Se han detectado algunos pliegos erróneamente determinados entre los asignados inicialmente a esta especie. Se trata de los pliegos VIT 75493 y VIT 75494, con material procedente de Portugalete (Vizcaya), que en realidad corresponden a la especie Ambrosia artemisiifolia. Lo mismo sucede con el pliego SANT 44001, de Rianxo (Coruña), asimismo perteneciente a esta última especie y no a Ambrosia tenuifolia.

Las siguientes referencias de herbario ayudan a establecer el área de la especie en España: Barcelona: Avinyonet del Penedès, 31TCF9979, 13-IX-1998, A. Sánchez Cuxart, MAF 158330. CÁdIZ: El Puerto de Santa María, 22-VI-1982, J. Devesa \& S. Talavera, SEV 79654. CanTabria: Santoña, 30TVP6010, 15-IX-1987, S. Patino \& J. Valencia, VIT 1541. VIzCAYA: Portugalete, 30TVN9795, 1-XI-1995, J. Elorza \& J. Valencia, VIT 35249.

Claves de determinación. El estudio morfológico y micromorfológico de las seis especies del género Ambrosia presentes en la Península Ibérica, ha conducido a la elaboración de unas claves de determinación que permiten identificar claramente dichas especies. Una de estas claves se basa preferentemente en caracteres foliares, mientras que la segunda atiende a aspectos relativos a las flores y los frutos. 

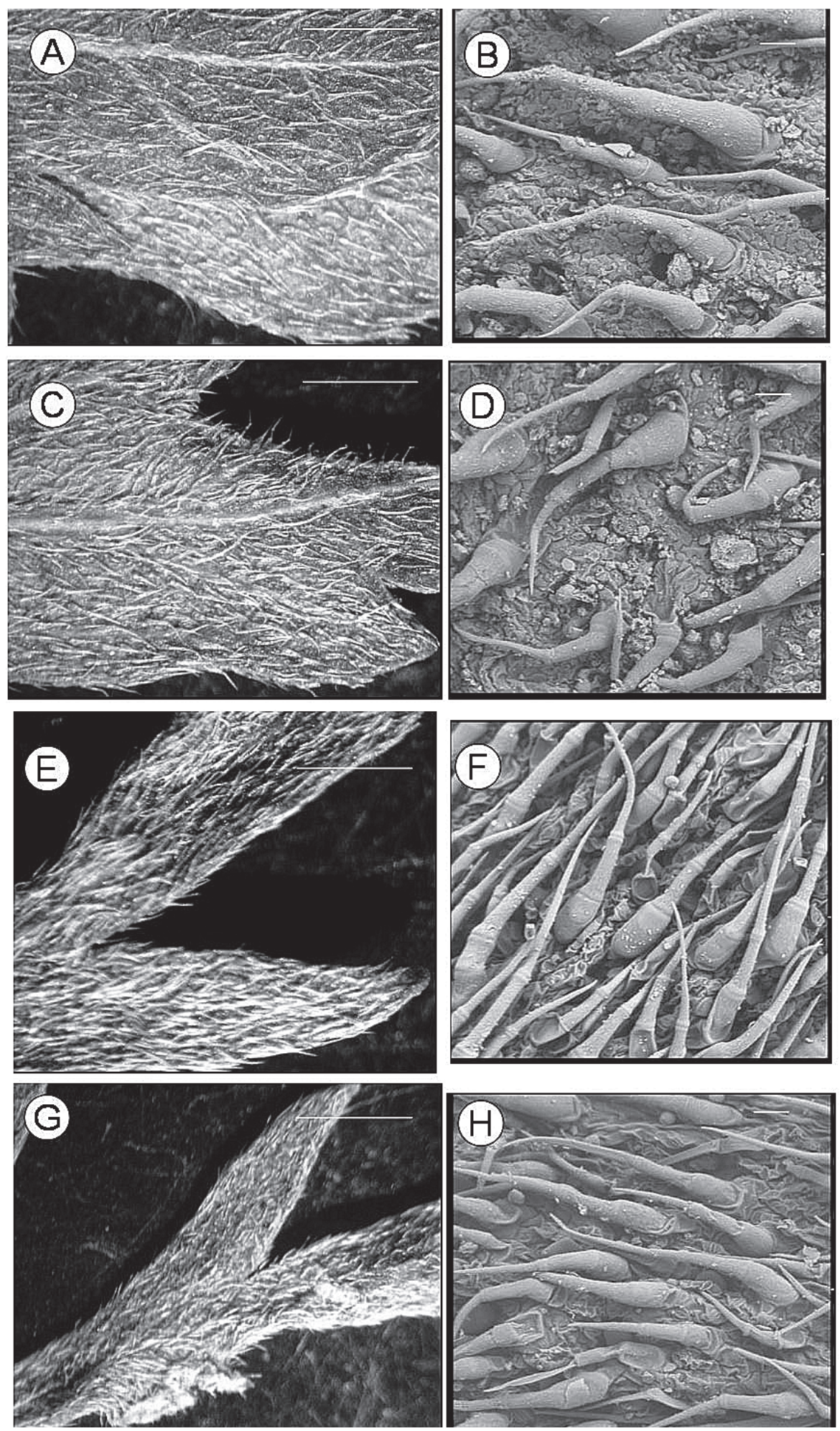

Fig. 4- Ambrosia psilostachya DC. A-B: cara adaxial de la hoja. C-D: cara abaxial de la hoja. Barra de escala: A-C: $1 \mathrm{~mm}$; B-D: $50 \mu \mathrm{m}$. A. tenuifolia L. E-F: cara adaxial de la hoja. G-H: cara abaxial de la hoja. Barra de escala: E-G: $1 \mathrm{~mm} ; \mathbf{F}-\mathbf{H}: 50 \mu \mathrm{m}$. 

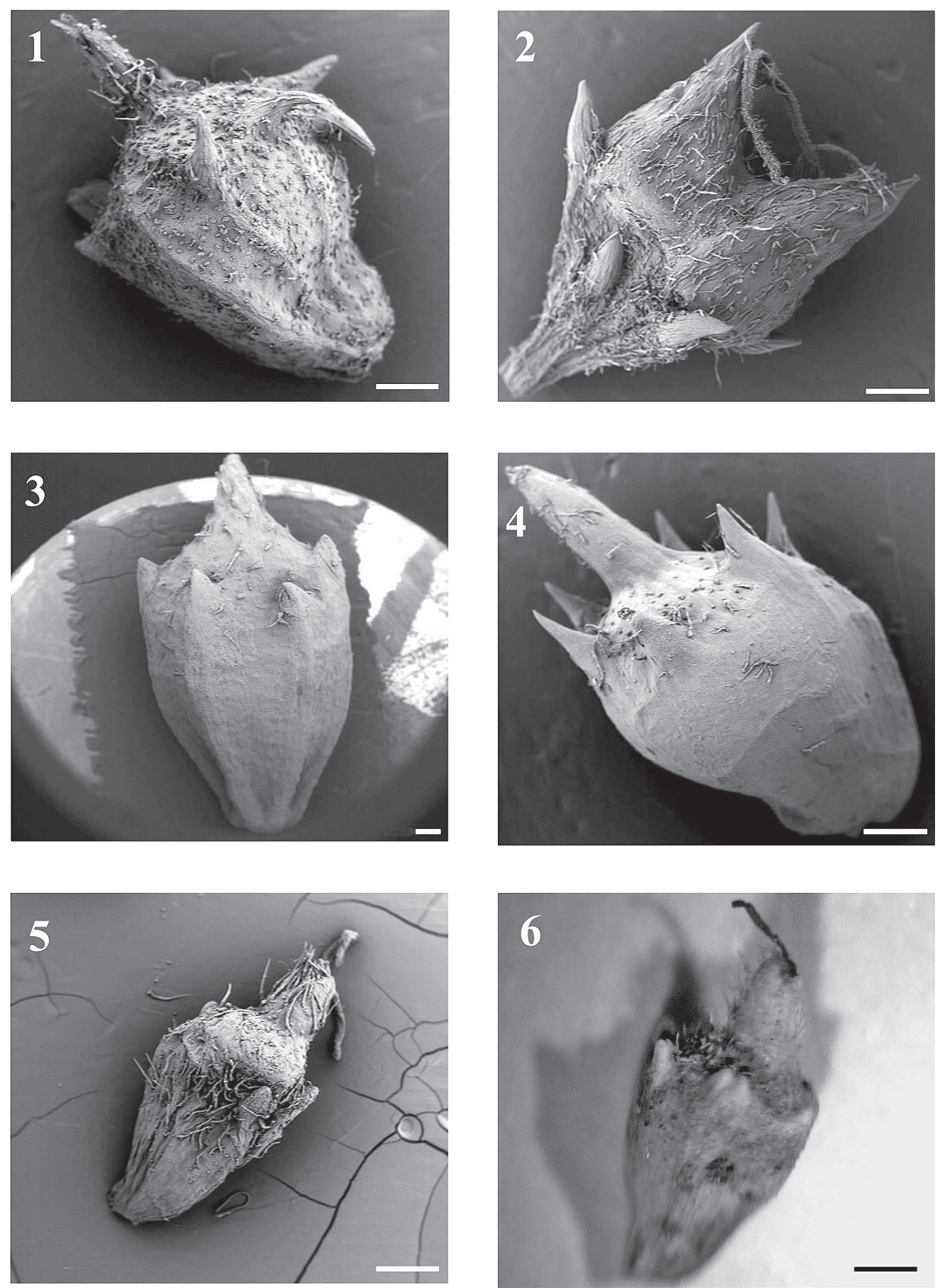

Fig. 5- Fotografías de las fructificaciones. 1. Ambrosia maritima. 2. A. tomentosa. 3. A trifida. 4. A. artemisiifolia. 5. A. psilostachya. 6. A. tenuifolia. Barra de escala: $0,5 \mathrm{~mm}$. 


\section{Clave basada en caracteres foliares (Fig. 6)}

1. Hojas inferiores bipinnatisectas, discoloras, cara adaxial verde cenicienta, cara abaxial blanquecina con un tomento de pelos denso.

1. Hojas inferiores enteras, palmeadas, con 3 ó 5 lóbulos o bipinnatisectas con lóbulos lineares, concoloras o ligeramente discoloras

2. Planta aromática, anual o bienal, con pelos largos intercalados con otros mas pequeños, excrecencias salinas, propia de arenales marítimos Ambrosia maritima

2. Planta no aromática, perenne, con tomento adpreso, rígido, blanquecino y denso, propia de hábitat terrestres del interior

Ambrosia tomentosa

3. Hojas opuestas, enteras o palmeadas, con 3 o 5 lóbulos de margen finamente serrado, escabras, pustulosas y pubescentes por ambas caras, con pequeños pelos estrigosos de base bulbosa acompañados de otros cortos unicelulares dispersos, que en la cara abaxial se localizan fundamentalmente en los nervios y dispersos por el haz. Peciolo alado de $3-12 \mathrm{~cm}$ de longitud..

Ambrosia trifida

3. Hojas alternas, opuestas o subopuestas, pinatipartidas o bipinnatisectas, con un corto peciolo alado o con un largo peciolo áptero, con pelos estrigosos de base bulbosa acompañados de otros pelos largos y articulados. ... 4

4. Plantas anuales, hojas subglabras o pubérulas por ambas caras, con pelos multicelulares escábridos de base bulbosa, mas densos en la cara abaxial.

4. Plantas perennes, hojas pubescentes con pelos aplicados cortos, rígidos y estrigosos por ambas caras Ambrosia artemisiifolia

5. Hojas subsésiles o con un corto peciolo alado, pinnadas, con lóbulos oblongos ..................................... Ambrosia psilostachya

5. Hojas pecioladas, peciolo áptero, pinnatipartidas y bipinnatisectas, con lóbulos lineares, generalmente dentados

Ambrosia tenuifolia

\section{Clave basada en caracteres florales y carpológicos}

1. Involucro femenino con 2 flores, generalmente con 2 aquenios, fructificación rematando en dos picos ... Ambrosia tomentosa

1. Involucro femenino con una flor única, generalmente con un solo aquenio, fructificación rematando en un pico ..

2. Fructificación generalmente tan larga como ancha, pubescente glandulosa, provista de 5 costillas bien marcadas, 5 tuberosidades cónicas en la parte media y terminando en un pico con 5 pequeños dientes ........................................... Ambrosia maritima

2. Fructificación generalmente mas larga que ancha, de pubescente glandulosa a glabra, con (4)5-6(7) dientes espinosos situados en la mitad superior, 0 bien inerme 0 con un verticilo formado por 4 dientes muy cortos

3. Involucro fructífero con 4 tuberosidades muy cortas (excepcionalmente inerme).

Ambrosia psilostachya

3. Involucro fructífero con (4)5-6(7) dientes espinosos o con otras tantas tuberosidades situadas en la mitad superior de la fructificación.

Ambrosia tenuifolia

4. Capítulos femeninos generalmente solitarios, raramente geminados

4. Capitulos femeninos agrupados en glomérulos axilares. ..... 5

5. Capítulos masculinos con involucro cupuliforme y un poco asimétricos, casi glabros, pelos multicelulares cortos y rígidos con base bulbosa, aquenio terminado en un pico cónico.

Ambrosia trifida

5. Capítulos masculinos turbinados, mas o menos pubescentes y ciliados, con pelos multicelulares cortos de base cónica y pelos largos, aquenio rematado en un pico provisto de cinco pequeños dientes.. Ambrosia artemisiifolia

\section{CONCLUSIONES}

Teniendo en cuenta el grave problema que supone la introducción de especies exóticas en la flora de un territorio, se ha elegido un grupo de neófitos perteneciente al género Ambrosia, cuya presencia en la Península Ibérica viene a ampliar el ya extenso catálogo de plantas alóctonas invasoras. Se trata de seis táxones: Ambrosia tomentosa, A. trifida, A. artemisiifolia, A. psilostachya, A. maritima y A. tenuifolia. Con la excepción de Ambrosia maritima, que se considera autóctona de la región Mediterránea, las otras cinco especies citadas son neófitos de origen americano que han ido estableciéndose en la península. El estudio corológico ha revelado que su área de distribución actual está aún ligada a las provincias de los litorales mediterráneo y atlántico por las que se ha producido su invasión, lo que se ve favorecido además por el clima más benigno de estas zonas. En algunos casos particulares, como los de Ambrosia artemisiifolia y A. tomentosa, su presencia ya ha sido puesta de manifiesto en territorios interiores peninsulares. 

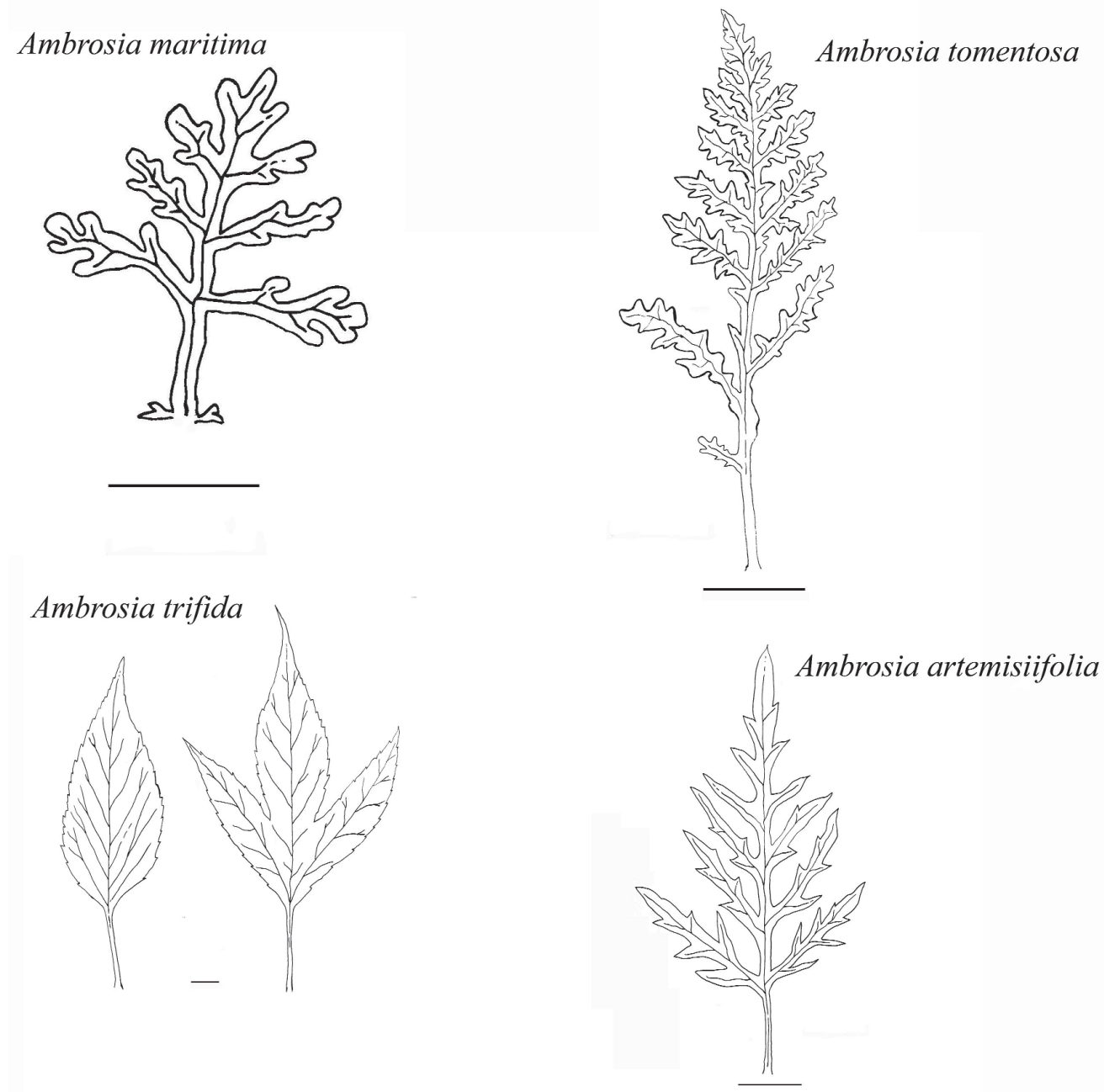

Ambrosia psilostachya
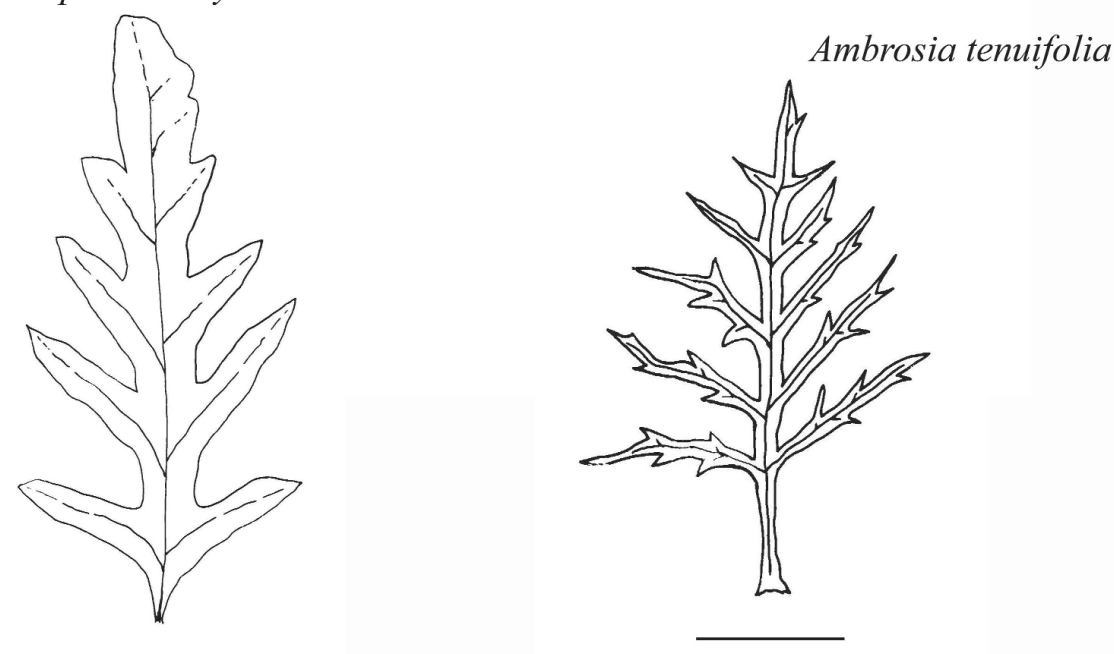

Fig. 6- Esquema de las hojas de las especies estudiadas. Barra de escala: $1 \mathrm{~cm}$. 
En cuanto a su ecología, se ha comprobado que son especies cuya introducción se ve favorecida por el aumento de las áreas antropizadas y alteradas, colonizando hábitats como cunetas de carreteras, vías férreas, cultivos abandonados y orillas nitrificadas de ríos y arroyos. En estos medios llegan a formar poblaciones muy densas que ponen en peligro determinadas especies y hábitats autóctonos.
El estudio morfológico y micromorfológico de hojas y frutos ha revelado que existen caracteres fácilmente observables como la forma, tamaño, pilosidad, color, etc., que ayudan a diferenciar claramente estas seis especies entre sí. Se han elaborado dos claves basadas en los datos de dicho estudio con el fin de proporcionar un instrumento útil para identificar estos neófitos que ya se pueden considerar como parte de la flora ibérica.

\section{BIBLIOGRAFIA}

Amaral Franco, J. Do. 1984. Nova Flora de Portugal (Continente e Açores). Lisboa.

Amor, A.; Navarro, F.; SÁnchez Anta. Ma ${ }^{a}$ A. \& Valle, C. J. 2007. Ambrosia artemisiifolia L. en la provincia Mediterránea Ibérica Occidental. Stud. Bot. 25: 133-136.

ANTHos. http://www.anthos.org. Real Jardín Botánico, CSIC, Madrid.

BeHÇET, L. 2004. A new record for the Flora of Turkey: Ambrosia tenuifolia Spreng. (Compositae). Turk J. Bot. 28: 201-203.

BoIsSIER, E. 1875. Flora Orientalis, 3. Ed. Asher \& Co B. V. Amsterdan 1975.

Bolós, O. \& Vigo, J. 1995. Flora dels Països Catalans 3: 769771. Ed. Barcino.

Borja Carbonell, J. 1951. Estudio fitográfico de la Sierra de Corbera (Valencia). Anales Jard. Bot. Madrid 9: 361-483.

Carretero, J. L. 1990. Aportaciones a la flora exótica española. Folia Bot. Misc. 7: 55-58.

Coste, H. J. 1900-1906. Flore descriptive et illustrée de la France, de la Corse et des contrées limitrophes. Paris.

CRONQUIST, A. 1994. Intermountain flora vascular plants of the intermountain West, U.S.A. 5. Asterales. The New York Botanical Garden, Bronx, New York.

Fireman, P. 2007. Atlas de alergia e inmunología clínica. Elsevier, España.

FolCH GuILLĖN, R. 1976. Notes floristiques, III. Quelques espèces nouvelles ou intéressantes de la zone littorale de la $\mathrm{Ca}$ talogne meridionale. Collect. Bot. (Barcelona) 10: 181-190.

Galdego, Ma . J. \& VAldÉs, B. 1984. Notas breves: Ambrosia temuifolia Spreng. Lagascalia 12(2): 270.

García Martínez, X. M. 1985. Algunas aportaciones a la flora gallega. Anales Jard. Bot. Madrid 42(1): 191-196.

Jovet, P. \& VILMORIn, R. 1975. Flore descriptive et illustrée de la France par H. Coste, 3eme. Suppl.: 299-300.

Laínz, M. \& LORIENTE, E. 1983. Contribuciones al conocimiento de la flora montañesa, II. Anales Jard. Bot. Madrid 39(2): 405-416.

LAmBinon, J. 1989. Bulletin de la Societè pour l'echange des plantes vasculaires de l'Europe et du bassin mediterraneen. Bélgica.

Linneo, C. 1753. Species Plantarum, 2. Estocolmo.

Masalles, R. M.; Sans, F.; Pino, J. \& Chamorro, L. 1996. Aportacions al coneixement de la flora sinantròpica catalana. Folia Bot. Misc. 10: 77-84.
MontserRat, P. 1954. La Ambrosia tenuifolia Spreng. en España. Collect. Bot. (Barcelona) 4: 311-313.

MonTSERRAT, P. 1962. Flora de la cordillera litoral catalana (porción comprendida entre los ríos Besòs y Tordera). Collect. Bot. (Barcelona) 6: 1-48. Barcelona.

PéreZ LaRA, J. M. 1887. Florula gaditana. Pars secunda. Anales Soc. Esp. Hist. Nat. 16(2): 273-372.

Pinto Da Silva, A. P. 1972. Treze espécies e subespécies novas para a flora de Portugal. Agronomia Lusitana 33: 1-24.

Rico HeRnÁNDEZ, E. 1981. Notas corológicas. Anales Jard. Bot. Madrid 38(1): 307-309.

Sanz Elorza, M.; Dana, E. \& Sobrino, E. 2001. Aproximación al listado de plantas alóctonas invasoras reales y potenciales de España. Lazaroa 22: 121-131.

Sanz ElorZa, M.; Dana, E. \& Sobrino, E. 2004. Atlas de las plantas alóctonas invasoras en España. Madrid.

Sanz Elorza, M.; González Bernardo, F. \& Serreta Oliván, A. 2009. La flora alóctona de Aragón (España). Bot. Complut. 33: 69-88.

Segura ZubiZARRETA, A. 1982. De flora soriana y otras notas botánicas (II). Homenaje almeriense al botánico Rufino Sagredo: 141-146. Instituto de Estudios Almerienses.

SmoLjaninova, L. A. 1999. Ambrosia L. En B. K. Schischkin (Eds.), Compositae, Flora of the USSR, 25: 1-574.

Trigo, Ma. M. \& García-SÁnchez, J. 2006. Ambrosia artemisiifolia L., nueva especie para la flora alóctona invasora de Andalucía (España). Acta Bot. Malacitana 31: 203-205.

Tutin, T. G. \& al. (Eds.) 1976. Flora Europaea, 4: 1-464.

Valdés, B.; Talavera, S. \& Fernández-Galiano, E. (Eds.) 1987. Flora vascular de Andalucía occidental, 3. Ed. Ketres. Barcelona.

Vallès Xirau, J. \& Poch Ymbert, J. M. 1999. Notes sobre algunes plantes al-lòctones a les comarques gironines $(\mathrm{Ca}$ talunya). Butll. Inst. Catalana Hist. Nat., Secc. Bot. 67: 62-65.

VAYReDA VILA, E. 1901. Notas geográfico-botánicas. Anales Soc. Esp. Hist. Nat. 29(3): 363-384.

VERLOOVE, F. 2005. New records of interesting xenophytes in Spain. Lazaroa 26: 141-148.

WillKomm, M. \& Lange, J. 1870. Prodromus Florae Hispanicae, 2. Stuttgart. 